Revista Electrónica de Investigación en Ciencias Económicas

Abriendo Camino al Conocimiento

Facultad de Ciencias Económicas, UNAN-Managua

Estrategias de vida campesina en cuatro comunidades ubicadas en áreas protegidas:

Una cosmovisión económica más allá de la agricultura

José Ramón Velásquez Hernández

Antropólogo Social, Máster en

Desarrollo Rural/UNAN-Managua

nicarao@hotmail.es

Fecha recepción: nov. / 12 del 2015

Fecha aceptación: dic. / 5 del 2015

Palabras claves: Estrategias de vida, cultura productiva, pluriactividad, unidad

doméstica campesina, área protegida

Key words: Livelihoods, productive culture, pluriactivity, peasant household, protected area.

ISSN: $2308-782 X$

\section{REICE}

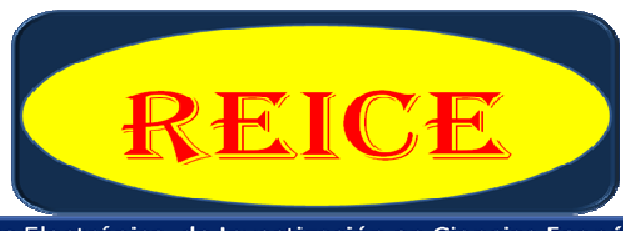

Revista Electrónica de Investigación en Ciencias Económicas

http://revistacienciaseconomicas.unan.edu.ni

revistacienciaseconomicas@gmail.com revistarucfa@unan.edu.ni

\section{Resumen}

En este estudio se analizan los tipos de estrategias de vida que desarrollan cuatro las comunidades campesinas ubicadas en las áreas protegidas de El Chocoyero-El Brujo y Miraflor-Moropotente. Para la realización del estudio se aplicaron encuestas que permitieron obtener información concreta sobre la forma en cómo se van configurando y adaptando a las condiciones variantes del entorno estas estrategias de vida. El $63 \%$ de las unidades domésticas campesinas viven de la agricultura y el restante $37 \%$ viven además de la agricultura de otras actividades económicas no agrícolas que son complementarias. De manera que la reproducción social de las UDC, no es exclusivamente en el marco de la agricultura, sino de otras actividades que acompañan. El marco interpretativo con el que se discuten teóricamente los hallazgos es la nueva ruralidad, y disertaciones teóricas sobre las estrategias de vida. La cultura campesina, no es sinónimo de lo agrario, existen componentes de la cultura rural que son más amplios y que determinan patrones de comportamientos específicos, como sus vínculos con lo urbano que influyen decididamente en la adopción de otras estrategias de vida que no son propias del contexto rural

\section{Abstract}

In this study the types of livelihood strategies that put forward four rural communities located in protected areas Chocoyero-El Brujo and MiraflorMoropotente are analyzed. For the study surveys that allowed specific information on the way how they are configured and adapted to the varying conditions of life environment these strategies were applied. $63 \%$ of rural households live from agriculture and the remaining $37 \%$ in addition to agriculture of other nonagricultural economic activities that are complementary living. So the social reproduction of the UDC, is not exclusively in the context of agriculture, but also other accompanying activities. The interpretive frameworks that theoretically discuss the findings is the new rurality, and theoretical lectures on the life strategies. The peasant culture is not synonymous with agriculture, there are components of rural culture which are broader and determine specific patterns of behavior, their links with urban influence decisively in the adoption of other livelihood strategies that are not proper the rural context. 
Revista Electrónica de Investigación en Ciencias Económicas

Facultad de Ciencias Económicas, UNAN-Managua

Estrategias de vida campesina en cuatro comunidades ubicadas en áreas protegidas: Una cosmovisión económica más allá de la agricultura

\section{Introducción}

El estudio sobre las estrategias de vida campesina, se realizó en dos áreas protegidas de Nicaragua. El primer contexto fue en la zona de amortiguamiento de El Chocoyero-Brujo, ubicado en el municipio de Ticuantepe, departamento de Managua, de la cual se seleccionaron a propósito de este artículo, dos comunidades, Los Ríos y La Francia 2, y el segundo contexto fue Miraflor-Moropotente, ubicado en el municipio de Estelí, departamento de Estelí, que constituye un paisaje terrestre protegido, se seleccionaron las comunidades de: Puertas Azules y La Fortuna-El Apante. El propósito central que persigue este artículo es analizar los tipos de estrategias de vida que desarrollan las comunidades antes mencionadas.

El análisis clásico esgrimido por Chayanov(1970), Wolf (1971), Fromm y Maccoby (1990) sobre la vida campesina, en donde el motor económico de la unidad doméstica se centraba en la agricultura, ha sido superado por la dinámica misma de las sociedades rurales, el campesino no solamente es productor agropecuario, sino que se desenvuelve en el contexto de la pluriactividad, Ferrus Batista(1988) de Janvry y Sadoulet(2000), Teubal(2012) Suarez y Tobasura(2008), Lanzas y Rojas(2011), Méndez, et. al(2012), el campesino es también obrero, guía turístico, artesano, comerciante (Kay, 2007) entre otras actividades que se van desarrollando en base a la necesidad de ingresos que tiene la familia campesina. Dichas estrategias son también el resultado del difícil acceso a los recursos naturales que tienen los comunitarios de las reservas naturales, en particular, lo que pone en el tapete de la realidad social, las desigualdades entre territorios ILPES(2012).

También se debe mencionar un estudio clásico, abordado desde la antropología pero que conecta precisamente con las realidades rurales, y es el estudio denominado Mujeres, graneros y capitales: economía doméstica y capitalismo (Meillassoux, 1989), en donde se planeta: "La comunidad doméstica es el único sistema económico y social que dirige la reproducción física de los individuos, la reproducción 
Revista Electrónica de Investigación en Ciencias Económicas

Facultad de Ciencias Económicas, UNAN-Managua

Estrategias de vida campesina en cuatro comunidades ubicadas en áreas protegidas: Una cosmovisión económica más allá de la agricultura

de los productores, y la reproducción social en todas sus formas, mediante un conjunto de instituciones". (pág. 9).

Se hace colación a esta obra clásica porque estos mecanismos de reproducción están presentes en las sociedades campesinas que se están estudiantes, en donde sus instituciones culturales son las encargadas de estos mecanismos de reproducción, y en este marco operan las estrategias de vida, con la única diferencia que estas unidades operan en contexto ecológico importante.

Agregar que para la comprensión, del funcionamiento de las unidades domésticas campesinas es necesario a los estudios clásicos de (Chayanov, 1981) sobre las sociedades agrarias, en donde plantea que: "el grado de explotación lo determina un equilibrio peculiar entre satisfacción de la demanda familiar y fatiga del trabajo" (pág. 54)

Las unidades domésticas campesinas tienen sus mecanismos de reproducción interna, y se está de acuerdo con Chayanov que la satisfacción de sus necesidades están en consonancia con el trabajo realizado esta, pero también se considera que el grado de satisfacción de estas necesidades también determinan la interacción con el entorno ecológico.

La diversificación de estas estrategias es el resultado de las dificultades que tienen los campesinos de acceder a la tierra, al financiamiento, a las tecnologías agrícolas, a mercados, entre otros factores internos y externos que intervienen en los territorios.

Un estudio novedoso que ha sido una referencia para los estudios sobre estrategias de vida en es el que titula: sustainable rural livelihoods: practical concepts for the 21st century publicado por (Chambers \& Conway, 1991). 
Revista Electrónica de Investigación en Ciencias Económicas

Facultad de Ciencias Económicas, UNAN-Managua

Estrategias de vida campesina en cuatro comunidades ubicadas en áreas protegidas: Una cosmovisión económica más allá de la agricultura

A livelihood comprises the capabilities, assets (stores, resources, claims and access) and activities required for a means of living; a livelihood is sustainable which can cope with and recover from stress and shocks, maintain or enhance its capabilities and assets, and provide sustainable livelihood opportunities for the next generation; and which contributes net benefits to other livelihoods at the local and global levels and in the short and long-term." (pág. 6)

Las estrategias de vida campesina se van adaptando a las circunstancias y condiciones de un entorno socio-cultural, económico, político y medioambiental complejo y que cambia constantemente. Una vez analizadas algunas características socioeconómicas como: la educación, la salud, los servicios básicos con que cuentan las unidades domésticas campesinas, es necesario hacer un análisis detallado de las estrategias de vida, las implicancias que estas tienen en la calidad de vida de sus miembros, y consecuentemente reflexionar qué perspectivas de desarrollo rural se vienen perfilando desde las unidades domésticas hacia la comunidad y el territorio.

El paradigma utilizado en el que se pretende englobar todo el contexto de las estrategias de vida campesina es una nueva ruralidad. En relación a esto También (De Grammont, 2008) en la definición del concepto de la Nueva Ruralidad analiza lo siguiente:

La nueva ruralidad, es entonces, una nueva relación campo-ciudad, en donde los límites entre ambos ámbitos de la sociedad se desdibujan, sus interconexiones se multiplican, se confunden y se complejizan. Existen dos grandes enfoques para el estudio de la nueva ruralidad, por un lado en que se centra en las transformaciones económicas, sociales y políticas de la sociedad. Por otro lado el dedicado a establecer cuáles deben ser las nuevas políticas públicas para responder no sólo a las actuales situaciones existentes en el campo, (producción agrícola, manufactura a domicilio, maquiladoras, pobreza, migración, etc), sino para que este cumpla con todas las funciones hoy que se le atribuyen: en Europa se enfatiza la conservación del 
Revista Electrónica de Investigación en Ciencias Económicas

Facultad de Ciencias Económicas, UNAN-Managua

Estrategias de vida campesina en cuatro comunidades ubicadas en áreas protegidas: Una cosmovisión económica más allá de la agricultura

medioambiente, en América Latina se busca fomentar un desarrollo equitativo. (pág. 25 y 26).

Por esa razón se plantean y se encuentran una serie de indicadores que reflejan que la agricultura no es la única estrategia de vida, y que los vínculos con los mercados y con lo urbano son fundamentales, la nueva ruralidad es el marco interpretativo de todo este análisis.

\section{Material y método}

El tipo de investigación es el no experimental, porque este sentido el investigador no manipula variables, sino que las variables sociales, culturales y económicas ya están establecidas. Se utilizan las entrevistas, grupos focales y observaciones como técnicas centrales y la encuesta como método auxiliar cuantitativo.

En relación con el método cualitativo (Barrantes, 2008) conceptualiza: "La investigación cualitativa postula una concepción fenomenológica, inductiva, orientada al proceso, busca dirigir o generar teorías. Pone énfasis en la profundidad y sus análisis no necesariamente son traducidos a términos matemáticos” (pág. 71).

En esta fase predomina la investigación documental en cuanto a la revisión de la bibliografía para construir los aspectos teóricos y metodológicos.

De acuerdo a (Alfonso, 1995) citado por (Morales, 2003) la investigación documental es: "Un procedimiento científico, un proceso sistemático de indagación, recolección, organización, análisis e interpretación de información o datos en torno a un determinado tema. Al igual que otros tipos de investigación, éste es conducente a la construcción de conocimientos" (Morales, 2003, pág. 2) 
Revista Electrónica de Investigación en Ciencias Económicas

Facultad de Ciencias Económicas, UNAN-Managua

Estrategias de vida campesina en cuatro comunidades ubicadas en áreas protegidas: Una cosmovisión económica más allá de la agricultura

Se parte del principio que no se puede desarrollar una investigación científica sin la previa documentación, por eso la investigación documental, es y constituye el punto de partida de toda investigación para la teorización del problema de estudio, en donde se siguieron los siguientes pasos: se ubica la literatura, se revisa, se selecciona, se analiza y se aplica a la temática estudiada.

Población y selección de la muestra a partir de un criterio estadístico ${ }^{1}$

Las cuatro comunidades que forman parte de la zona de amortiguamiento del refugio de vida silvestre El Chocoyero-El Brujo, tienen una población de 1,770 habitantes según INIDE(2005), en donde Los Ríos y San José es visto como una sola comunidad, pero en realidad están divididas; la Francia 2 tiene una población de 51 , y La Francia 1, de 32 pobladores.

Tipo de muestra, se utiliza la fórmula de Fisher para extraer la muestra en las cuatro comunidades de la zona de amortiguamiento de El Chocoyero-El Brujo.

$$
\frac{Z^{2} * p^{*} q^{*} N}{\left(N^{*} e^{2)}+\left(Z^{2} * p^{*} q\right)\right.}
$$

$\mathrm{Z}=1.6449$

$\mathrm{N}=1,770$.

p y $q=0.5 \times 0.5$

$\mathrm{e}=0.0875$

$\mathrm{n}=84$

La aplicación de la formula indica una muestra de 84 y con el fin de ajustar el tamaño muestral previsto para el estudio a posibles pérdidas de información que se produzcan durante el desarrollo del mismo. Así, asumiendo un porcentaje del $20 \%$ de pérdidas, el tamaño de la muestra ajustada será de 105.

\footnotetext{
${ }^{1}$ Se hizo el cálculo a partir de la fórmula para poblaciones finitas, tomando en consideración las ocho comunidades rurales estudiadas, cuatro en El Chocoyero-El Brujo y cuatro en Miraflor-Moropotente, para este estudio se seleccionaron cuatro comunidades rurales, dos por cada territorio.
}

REICE Vol. 3, No. 6, julio-diciembre 2015

ISSN: $2308-782 X$ 
Revista Electrónica de Investigación en Ciencias Económicas

Facultad de Ciencias Económicas, UNAN-Managua

Estrategias de vida campesina en cuatro comunidades ubicadas en áreas protegidas: Una cosmovisión económica más allá de la agricultura

Las cuatro comunidades que forman parte de la zona núcleo del paisaje terrestre protegido Miraflor Moropotente, tienen una población de 7,500 habitantes según (Procuraduría General de la República, 2008), en donde se seleccionaron cuatro comunidades para la realización del el estudio.

REICE $\mid 61$

Tipo de muestra, se utiliza la fórmula de Fisher para extraer la muestra en las cuatro comunidades de Miraflor-Moropotente.

$$
\frac{Z^{2} * p^{*} q^{*} N}{\left(N^{*} e^{2)}+\left(Z^{2} * p^{*} q\right)\right.}
$$

$\mathrm{Z}=1.6449$

$\mathrm{N}=7,500$

p y $q=0.5 \times 0.5$

$\mathrm{e}=0.0875$

$\mathrm{n}=87$

La aplicación de la formula indica una muestra de 87 y con el fin de ajustar el tamaño muestral previsto para el estudio a posibles pérdidas de información que se produzcan durante el desarrollo del mismo. Así, asumiendo un porcentaje del 14\% de pérdidas, el tamaño de la muestra ajustada será de 101.

\section{Resultado y Discusión}

Diversificación de estrategias representadas en el tipo de empleo

Empezamos este análisis con las comunidades que conforman el territorio de amortiguamiento de la reserva El Chocoyero-El Brujo, y las comunidades que de la reserva Miraflor-Moropotente, al igual que el aspecto educativo, se van comparando comunidades de un contexto y otro.

\section{Comunidad de Los Ríos}

Se analizan las estrategias de vida, a partir del tipo de empleo que desempeñan los campesinos, y como estos están condicionados también por factores externos a la comunidad, además que la cercanía a núcleos urbanos tiene una incidencia significativa.

REICE Vol. 3, No. 6, julio-diciembre 2015

ISSN: $2308-782 X$ 
Revista Electrónica de Investigación en Ciencias Económicas

Facultad de Ciencias Económicas, UNAN-Managua

Estrategias de vida campesina en cuatro comunidades ubicadas en áreas protegidas: Una cosmovisión económica más allá de la agricultura

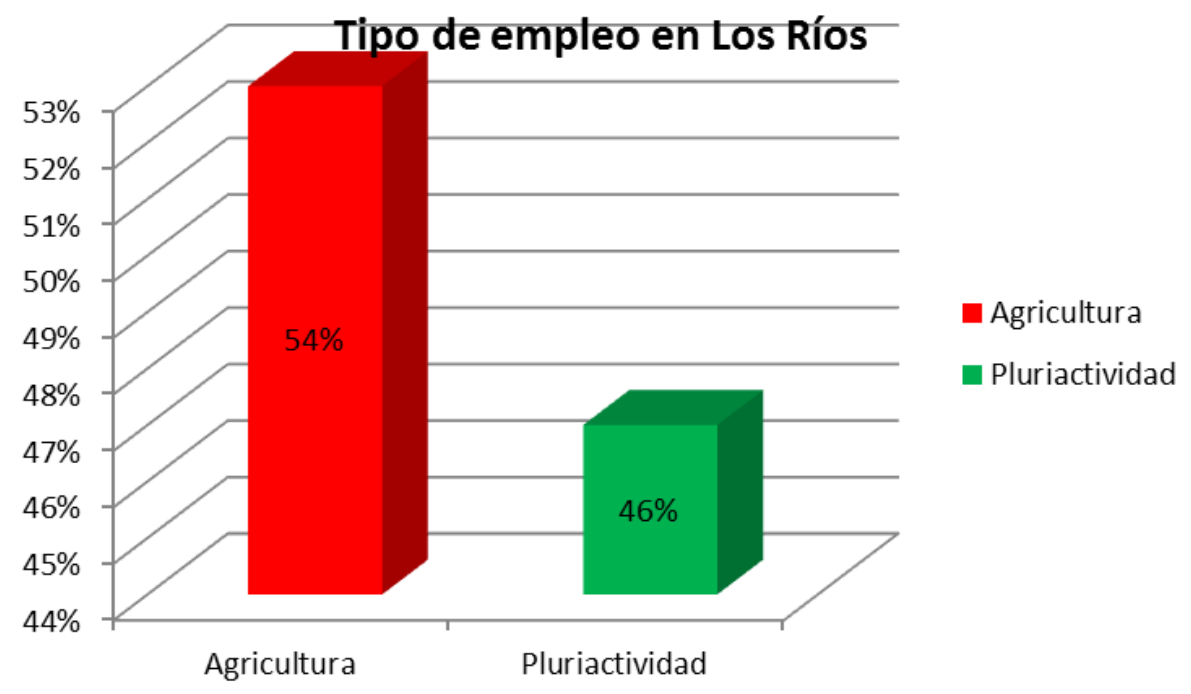

REICE $\mid 62$

Gráfico 1. Tipos de empleo en la comunidad de Los Ríos. Fuente propia.

De acuerdo a las encuestas aplicadas a 30 jefes de familias, se encontró que el 54\% se dedica únicamente a la agricultura, y el $46 \%$ diversifica sus actividades económicas para mejorar el ingreso en sus familias. Por un lado se considera que la agricultura debe ser el medio de subsistencia central en contextos rurales, para el fortalecimiento de la identidad productiva campesina, por otro dedicarse solamente a esta actividad limita el ingreso y no permite solventar las necesidades de la unidad doméstica.

Empero la diversificación de actividades económicas, es una estrategia que permite ampliar el horizonte productivo, y no dedicarse sólo a la agricultura, sino a otras actividades que inevitablemente apuntan a la tercerización de los servicios, y que van construyendo y reconfigurando una identidad diferenciada, la diversificación de estrategias se contempla en las siguientes actividades: obrero agrícola 10\%, amas de casa 7\%, lavar y planchar 3\% ajeno, negocio de mototaxi 3\%, guía turístico 3\%, agricultura y costurera 3\%, agricultura y obrero agrícola $7 \%$, agricultura, comerciante $3 \%$ y ama de casa $7 \%$, se observa que la mayoría de estas actividades se hacen acompañar de la agricultura.

REICE Vol. 3, No. 6, julio-diciembre 2015

ISSN: $2308-782 X$ 
Revista Electrónica de Investigación en Ciencias Económicas

Facultad de Ciencias Económicas, UNAN-Managua

Estrategias de vida campesina en cuatro comunidades ubicadas en áreas protegidas: Una cosmovisión económica más allá de la agricultura

Para el $54 \%$ de las unidades domésticas, la agricultura es suficiente para garantizar el funcionamiento y reproducción de las unidades domésticas, para el 46\%, la diversificación es la manera en que pueden funcionar, ya sea porque estas familias tienen escases de la tierra o porque ven la oportunidad de un ingreso mayor fuera de las explotaciones agropecuarias. Sin embargo se considera necesario diversificar las actividades económicas dentro de las unidades domésticas productivas, y no enfocarse únicamente en la agricultura, sin dejar de considerarla como la actividad principal de identidad productiva rural.

En cuanto a la reproducción de las unidades domésticas campesinas:

Las Unidades Domesticas Campesinas emprenden diversas estrategias para reproducirse socioeconómicamente en el entorno en el que se desenvuelven, ejecutando actividades agrícolas y ganaderas, así como también actividades no agrícolas que contribuyen a la formación de sus ingresos económicos para hacer frente a las necesidades que se generan como unidad de producción (Lanzas \& Rojas, 2011, pág. 95).

Se considera que las unidades domésticas campesinas van moldeando sus estrategias, conforme van surgiendo necesidades prioritarias en torno a sus familias, es un asunto de adaptabilidad y hasta cierto punto de resiliencia, porque una de los riesgos que se corren con la producción agrícola tiene que ver con las externalidades, sean estas las condiciones cambiantes del ambiente, como la sequía, o fuertes lluvias, o los bajos precios de los productos en el mercado.

Por eso es importante que la vía agrícola no constituya la única fuente de ingresos, sino que se deben diversificar las estrategias. Se considera que las mencionadas anteriormente no son las óptimas pero si las que están al alcance de las familias rurales, tomando por supuesto en consideración el análisis hecho en el capítulo anterior, sobre el alarmante problema educativo, que no permite visualizar 
Revista Electrónica de Investigación en Ciencias Económicas

Facultad de Ciencias Económicas, UNAN-Managua

Estrategias de vida campesina en cuatro comunidades ubicadas en áreas protegidas: Una cosmovisión económica más allá de la agricultura

estrategias más productivas, ecoamigables, que estén en pro de la conservación de la identidad cultural rural, y por consiguiente de los recursos naturales propios de la reserva.

Se compara la comunidad núcleo de El Chocoyero-El Brujo con Puertas Azules que es la comunidad núcleo de Miraflor-Moropotente.

\title{
Puertas Azules
}

La comunidad de Puertas Azules, a pesar de que vivió una contra reforma agraria, sigue siendo agrícola, sin embargo paulatinamente su población ha diversificado sus ocupaciones, debido a la necesidad de incrementar sus ingresos económicos.

\section{Tipo de empleo Puertas Azules}

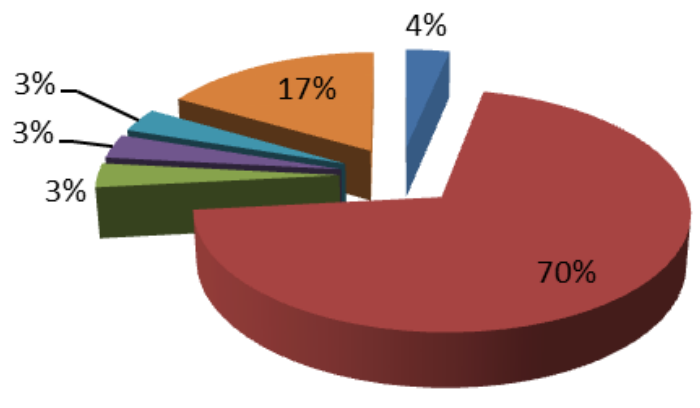

\author{
- Administrador de ONG \\ - Agricultura \\ - Agricultura y doméstica \\ - Agricultura y ganadería \\ - Comerciante y agricultor \\ - Obrero agrícola
}

Gráfica 2. Tipos de empleos en Puertas Azules. Miraflor. Fuente propia.

En puertas azules el $70 \%$ de las unidades domésticas campesinas, se dedican únicamente a la agricultura, de acuerdo a los resultados encontrados en una muestra de 30 familias, el peso está en esta actividad económica, aunque diversificada a través del cultivo de diversos rubros. El restante $30 \%$ ejercen la pluriactividad, es decir otras actividades que no necesariamente son la agricultura, no obstante 
Revista Electrónica de Investigación en Ciencias Económicas

Facultad de Ciencias Económicas, UNAN-Managua

Estrategias de vida campesina en cuatro comunidades ubicadas en áreas protegidas: Una cosmovisión económica más allá de la agricultura

pueden estar acompañadas de esta. Las unidades domésticas que están en el marco de la pluriactividad como alternativa están distribuidos de la siguiente manera: $4 \%$ administrador de ONG, 17\% obreros agrícolas, 3\% comerciante y agricultor, $3 \%$ agricultura y ganadería, $3 \%$ agricultura y doméstica.

REICE $\mid 65$

En Los Ríos hay mayor orientación hacia la pluriactividad, en relación al Puertas Azules, debido a las características espaciales de este contexto y su cercanía con Managua, lo que le imprime un carácter más vertiginoso en relación a la segunda comunidad abordada. Lo que debe mantener el interés en el estudio de estos procesos es el paulatino incremento de obreros agrícolas que trabajan en las fincas de la comunidad, y se enfrenta la comunidad rural a un proceso inminente de descampesinización, y por consiguiente el cambio de identidades productivas.

Al igual que el caso anterior, las actividades que conforman la pluriactividad, siempre van acompañadas de la agricultura, aunque trabajen en otras actividades no agrícolas, no dejan de practicar la agricultura, es necesario que en este panorama y analizando las tendencias mundiales, donde el agro está en manos de los grandes capitales y domina no solo la tierra, sino también la semilla y los agroquímicos, lo cual constituye un mercado importante, pero no sólo eso, bajo estas condiciones hay un inminente proceso de captación de fuerza de trabajo agrícola, y por lo tanto un creciente aumento de obreros agrícolas, que se han visto despojados de la propiedad (Teubal, 2001, pág. 46 y 52) y la agroindustria rural avanza rápidamente, y el proceso de transformación rural es ya un hecho social real, debido a las desigualdades en el acceso a recursos naturales tanto a lo interno de las unidades domésticas, como a nivel de la comunidad (Ferrus, 1988, pág. 127). En Puertas Azules, hay quienes todavía disponen de la tierra, y otros que no la poseen, debido a las condiciones adversas al entorno social, económico y político, y es por eso que las estrategias se están dirigiendo hacia otras actividades de tipo no agrícola. 
Revista Electrónica de Investigación en Ciencias Económicas

Facultad de Ciencias Económicas, UNAN-Managua

Estrategias de vida campesina en cuatro comunidades ubicadas en áreas protegidas: Una cosmovisión económica más allá de la agricultura

Las comunidades están interconectadas, no solamente entre estas, sino con territorios más amplios, que trascienden los limites nacionales, por el hecho de ser reservas, están bajo el lente de otros actores, agentes, organizaciones e instituciones internacionales. Por lo tanto los procesos de cambios culturales y sociales, las normas, hábitos, tradiciones y costumbres pueden transformarse de manera vertiginosa, es ahí donde se hacen necesarios mecanismos fuertes de cohesión socio-cultural.

En el marco de este análisis, Lanzas y Rojas(2011) analizan los mecanismos internos de reproducción de la unidad doméstica campesina en un primer momento, pero (Teubal, 2001) y (Ferrus, 1988), aportan las causas externas de la multifuncionalidad y la multiocupación, desde el mercado internacional, los organismos multinacionales y el acercamiento de la agroindustria a lo rural, es ahí donde las pobladores empiezan a ocuparse en las actividades extra agrícolas, eso en el caso de los países latinoamericanos, pero lo que no mencionan es que estas actividades son estimuladas por un creciente proceso de urbanización, las conexiones entre las comunidades rurales y los contextos urbanos, concretamente las cabeceras municipales hacen posible el desarrollo de las actividades no agropecuarias.

En Nicaragua no se puede hablar de un agresivo proceso de industrialización rural, pero sí de contextos urbanos cuya población crece demográficamente, y que demanda alimentos, a la vez que ejerce presión sobre la tierra, es ahí donde se ven obligados los pobladores rurales a aumentar sus áreas de cultivo, y la presión sobre los recursos de la reserva es aún mayor, además de la demanda de alimentos, el proceso de urbanización ejerce también una poderosa influencia, con el acercamiento constante hacia áreas de las reservas, entonces se tiene una doble presión sobre la tierra y sobre el territorio. 
Revista Electrónica de Investigación en Ciencias Económicas

Facultad de Ciencias Económicas, UNAN-Managua

Estrategias de vida campesina en cuatro comunidades ubicadas en áreas protegidas: Una cosmovisión económica más allá de la agricultura

A continuación se abordará la comunidad de La Francia 2, para comprender el territorio de amortiguamiento de la Reserva El Chocoyero-El Brujo.

\title{
La Francia 2
}

REICE | 67

Esta comunidad es eminentemente agrícola, sin embargo algunos de sus pobladores, están orientándose hacia otras actividades económicas como complemento de la actividad central.

\section{Tipo de empleo en La Francia 2}

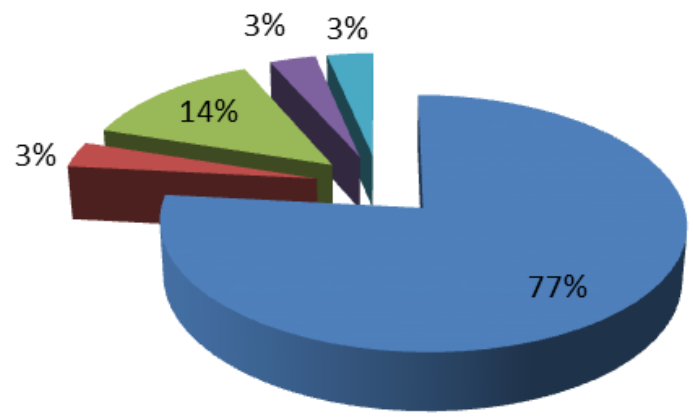

\author{
Agricultura \\ - Albañil \\ Ama de casa \\ Instituciones públicas \\ Maestro de Obra
}

Gráfica 3. Tipos de empleos en la Francia 2. Fuente propia.

De acuerdo a la información obtenida de los jefes de familias, el 77\% de estos se emplean en la agricultura familiar, el $3 \%$ es maestro de obra, el $3 \%$ trabaja en instituciones públicas, otro 3\% trabaja en construcción y el 14\% son amas de casa, por las construcciones socioculturales esta última actividad no se considera un trabajo como tal, pero se debe destacar que la amas de casa brindan un aporte importante a la economía familiar. Se debe destacar que en estas comunidades todas estas actividades extra agrícolas, van acompañadas de otras actividades complementarias incluyendo la agricultura. 
Revista Electrónica de Investigación en Ciencias Económicas

Facultad de Ciencias Económicas, UNAN-Managua

Estrategias de vida campesina en cuatro comunidades ubicadas en áreas protegidas: Una cosmovisión económica más allá de la agricultura

El peso porcentual en cuanto a la agricultura en Puertas Azules, se asemeja al de La Francia 2, con una ligera diferencia del 7\%, lo que indica que a pesar de la influencia urbana, esta comunidad tiene mucho más arraigada su vocación agrícola, también se debe destacar que esta agricultura es su mayor parte con fines comerciales, mediante la observación se ha logrado constatar las extensas plantaciones de piña en esta comunidad, que aunque no tiene un enfoque de seguridad alimentaria en la comunidad, representa una estrategia de vida para la población campesina.

En el marco del funcionamiento de las unidades domésticas campesinas, se debe considerar que estas estrategias forman parte de la resiliencia, es decir adaptarse a las condiciones variables del entorno, a través de la combinación de actividades agrícolas y extra agrícolas.

En esta misma dirección se señala:

Para hacer frente a las continuas variaciones de su entorno, los campesinos se ven obligados a crear y recrear permanentemente nuevas estrategias, a fin de alcanzar su reproducción social. Las estrategias campesinas pueden ser muy diversas, e incluir a actores sociales muy diferentes. Estas comprenden no sólo la producción predial y las actividades extra prediales, sino también todos aquellos procesos relacionados con la esfera doméstica (criar y educar a los hijos, cocinar, acarrear leña y agua, etc.). (Cáceres, 1995, pág. 70)

De acuerdo con el autor, la creatividad en cuanto a definir nuevas estrategias que le permiten a la unidad doméstica campesina su reproducción, sin embargo en los contextos de reserva natural, la necesidad obliga a buscar otras alternativas para complementar el ingreso, cómo por ejemplo trabajar de asistente del hogar (trabajo doméstico para otras familias) en el sector de la construcción o como obrero agrícola, y si esas estrategias se definen en el seno de las actividades agrícolas, y se cultivan rubros como la piña, no están estas estrategias en el marco de la sostenibilidad. 
Revista Electrónica de Investigación en Ciencias Económicas

Facultad de Ciencias Económicas, UNAN-Managua

Estrategias de vida campesina en cuatro comunidades ubicadas en áreas protegidas: Una cosmovisión económica más allá de la agricultura

De manera que los trabajos alternativos provocan hasta cierto punto una desidentización campesina, porque paulatinamente la población va perdiendo la vocación por la agricultura, y al paso de las generaciones, los valores y práctica sobre el trabajo en el campo, se van transformando, y si se habla de rubros rentables como estrategias, estos no cumplen con el criterio de sostenibilidad, porque el $77 \%$ que se dedica a la agricultura en su mayor parte son productores de piña, para su comercialización en los espacios de mercado.

En las tres comunidades que se han analizado hasta el momento, más del 50\% de su población tiene vocación agrícola, se puede decir que existe fortaleza en la identidad productiva y como práctica productiva, sin embargo ya la pluriactividad empieza a hacer contrapeso a la actividad principal, porque las familias deben cubrir sus necesidades, y para esto necesitan conexión con lo urbano.

En cuanto a las estrategias de vida, Mónica Vargas en un estudio realizado en México sobre Estrategias de sobrevivencia campesinas, plantea en las estrategias de sobrevivencias no agrícolas o urbanas:

El medio rural caracterizado por una planta productiva insuficiente y pobre y con una técnica atrasada y rudimentaria, se halla en contraposición al medio urbano, donde existen ciudades con industrias, comercios, servicios, centros culturales, entre otros, aspectos que hacen que las ciudades sean atractivas para la inserción laboral y para aumentar el nivel de vida del migrante rural, meta que generalmente nunca alcanza (Vargas, 1996, pág. 48).

Siguiendo la línea de pensamiento de esta investigadora, consideramos que los contextos urbanos, ejercen presión sobre lo que se debe consumir y sobre lo que se debe vender, esto también es vinculante con el mercado, sin embargo hay empleos propios de la dinámica de lo urbano como: trabajo doméstico, guardas de seguridad, 
Revista Electrónica de Investigación en Ciencias Económicas

Facultad de Ciencias Económicas, UNAN-Managua

Estrategias de vida campesina en cuatro comunidades ubicadas en áreas protegidas: Una cosmovisión económica más allá de la agricultura

trabajo de mototaxis, empleos en instituciones públicas, comercio, obreros agrícolas entre otros no menor importancia, que representan opciones que la misma dinámicas rural-urbano configura, en el caso de las comunidades estudiadas en El Chocoyero-El Brujo, los contextos urbanos de Ticuantepe y Managua, ejercen una influencia poderosa sobre las comunidades, y en el caso de las comunidades que se estudiaron en Miraflor-Moropotente, Yalí y Estelí constituyen los entornos urbanos de influencia. Se considera al igual que Palerm, A(2008) que el capitalismo ha logrado influenciar a los contextos rurales, más que en las propuestas de transformación de la fórmula general del capital M-D-M, ha transformado la cultura, los hábitos, las percepciones sobre lo rural, pero sobre todo en lo ecológico, el capitalismo con sus distintas variantes constituyen una amenaza para las reservas naturales, (Palerm, 2008).

Por un lado el capital extrae todo los recursos de las reservas, incluyendo el agua y los alimentos, a la vez que ejerce presión sobre los recursos, pero por otro el capitalismo de estado, ni la iniciativa privada ha logrado establecer en sus comunidades, servicios básicos elementales de calidad e infraestructura vial, por estas razones, y por la poca inversión en actividades propias de los contextos rurales, es que existe la precarización laboral, y la perpetuación de la línea de la pobreza en el campo.

\section{La Fortuna-El Apante}

Esta es una comunidad donde prevalecen medianas empresas agropecuarias, que emplean no sólo a los y las habitantes de esta comunidad, sino que emplea a pobladores de comunidades aledañas como: Puertas Azules, El Sijul entre otras, sin embargo estas empresas dotadas de un capital fuerte, coexisten con las pequeñas explotaciones agropecuarias de la comunidad. 
Revista Electrónica de Investigación en Ciencias Económicas

Facultad de Ciencias Económicas, UNAN-Managua

Estrategias de vida campesina en cuatro comunidades ubicadas en áreas protegidas: Una cosmovisión económica más allá de la agricultura

\title{
Tipos de empleos en La Fortuna-El Apante
}
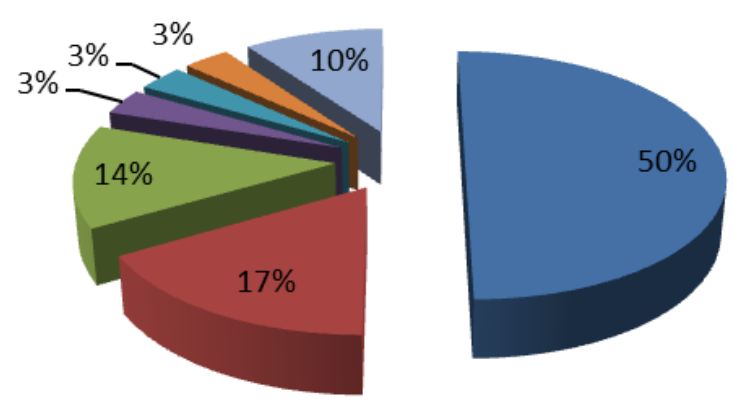

\author{
n Agricultura \\ - Agricultura y Ama de casa \\ Agricultura y Obrero \\ Agrícola \\ - Agricultura, Ama de casa \\ y Obrero Agrícola \\ Ama de Casa \\ Doméstica \\ - Obrero agrícola
}

REICE $\mid 71$

Gráfica 4. Tipos de empleos en la comunidad La Fortuna-El Apante. Fuente propia.

De acuerdo a la encuesta aplicada a 30 jefes de familia, en esta comunidad el $50 \%$ de las unidades domésticas campesinas se dedican principalmente a la agricultura, el $17 \%$ combinan entre agricultura y trabajo como ama de casa, en este caso particular se menciona cuando las mujeres son jefes de familia, $14 \%$ son jefes de familias y obreros agrícolas, un 10\% son obreros agrícolas, un $3 \%$ de las unidades domésticas combinan entre agricultura, ama de casa y obrero agrícola, un 3\% son amas de casa y otro $3 \%$ son domésticas(trabajadoras asistentes del hogar).

La observación directa, logró revelar que esta es una comunidad muy productiva, sin embargo las grandes explotaciones agrícolas están en manos de propietarios privados, que controlan propiedades de hasta 135 manzanas, contratan fuerza de trabajo de hasta 130 obreros y producen entre 80,000 y 100,000 quintales de papa,(esta información fue proporcionada por Santos Barreda, Daniel González y María Centeno, líderes comunitarios) es en este contexto donde el capitalismo agrario opera en contextos de reserva natural y donde no hay por consiguiente sustentabilidad. 
Revista Electrónica de Investigación en Ciencias Económicas

Facultad de Ciencias Económicas, UNAN-Managua

Estrategias de vida campesina en cuatro comunidades ubicadas en áreas protegidas: Una cosmovisión económica más allá de la agricultura

En relación a la contradicción capitalismo-sostenibilidad Elizalde propone:

El crecimiento capitalista se basa en la permanente creación de necesidades, muchas de ellas artificiales, para sostener la demanda de nuevos bienes necesaria para alimentar el sistema. La sociedad capitalista actual ancla su existencia en la producción industrial de bienes de consumo masivo, bienes que requieren ser permanentemente desvalorados y desechados, para así continuar creando nuevos bienes que los sustituyan (Elizalde, 1996).

Se toma como referencia está reflexión que hace Elizalde, precisamente una de las contradicciones que no revelaron los pensadores de pensamiento marxista, es la del capital con la naturaleza, con lo ecológico. El productor de papa, emplea cantidades considerables de agroquímicos para la producción, en primer lugar enriquece a los vendedores locales, nacionales y a las empresas transnacionales que venden estos tipos de agentes contaminantes, en segundo lugar contamina de manera sistémica(tierras, agua, recursos forestales, animales y seres humanos) que habitan en la reserva, y en tercer lugar tiene impuesta una necesidad de alimentar a masas de población considerable en la ciudades, obteniendo con su venta un beneficio económico.

A pesar de la inserción del sistema capitalista en la comunidad este coexiste con el sistema de producción campesino. "Pero el modo de producción capitalista, no es la única forma de producción en la sociedad moderna, pues conjuntamente con él vemos todavía vestigios, de otros sistemas de producción precapitalista conservados hasta nuestros días" (Kautsky, 1974, pág. 9).

Este subsistema que menciona el autor, es el reflejo de la existencia paralela de las pequeñas explotaciones agropecuarias y las empresas agrícolas, sin embargo se debe mencionar que estas estrategias no agrícolas son el reflejo de un sistema 
Revista Electrónica de Investigación en Ciencias Económicas

Facultad de Ciencias Económicas, UNAN-Managua

Estrategias de vida campesina en cuatro comunidades ubicadas en áreas protegidas: Una cosmovisión económica más allá de la agricultura

capitalista que profundiza las asimetrías entre el gran productor y los pequeños, pero

a cómo analiza Palerm:

En algunas ocasiones la venta por dinero de M' y de MT al área capitalista dominante sirve para efectuar procesos de reequipamiento de los medios de producción, que a veces aparecen como esfuerzos de capitalización incipiente. También sirven, con más frecuencia, para adquirir o rentar más tierras y aun para alquilar temporalmente fuerza de trabajo suplementaria. Es decir, en cualquier caso refuerzan el modo campesino de producción y no contribuyen directamente a su disolución. (Palerm, 2008, pág. 309)

Sin embargo las actividades no agrícolas como: ama de casa, doméstica, comerciante, entre otras, pueden ser un mecanismo para mantener cohesionada a la familia campesina y que persistan en áreas rurales a través de estas estrategias y de esta manera eviten su desplazamiento, pero también puede representar el inicio del mismo. La similitud de esta comunidad con La Francia 2 es que hay fortaleza en la agricultura familiar, y que diversifican sus estrategias con actividades extra agrícolas, también constituye un común denominador en todas las comunidades, la diferencia es que la agricultura en La Francia 2, está orientada al mercado, puede ser que de campesinos pasen a ser agricultores (Kautsky, 1974, pág. 16), y en el caso de La Fortuna-El Apante tiene un fuerte componente de obreros agrícolas. 
Revista Electrónica de Investigación en Ciencias Económicas

Facultad de Ciencias Económicas, UNAN-Managua

Estrategias de vida campesina en cuatro comunidades ubicadas en áreas protegidas: Una cosmovisión económica más allá de la agricultura

\section{Conclusiones}

Las estrategias de vida siguen teniendo un fuerte enfoque agrícola, sin embargo es notorio el avance de la pluriactividad que se encamina hacia la tercerización, muchas veces obedeciendo a dinámicas propias de las comunidades y en otras al mercado y a lo urbano, lo ecológico está siendo fuertemente influenciado por agricultura con fines comerciales en las comunidades que constituyen áreas protegidas.

El $63 \%$ de las unidades domésticas campesinas, desarrollan la agricultura como actividad principal, esto quiere decir que a pesar los planteamientos de la nueva ruralidad, esta actividad sigue siendo el motor principal de la economía comunitaria. Sin embargo el $37 \%$ de las UDC además de la agricultura, desarrollan otras actividades económicas para complementar sus ingresos.

La reproducción social de las UDC se desarrollan también en actividades complementarias a la agricultura, de manera que pensar en los territorios rurales como contextos que desarrollan únicamente actividades agrícolas, no es propio de estos nuevos tiempos, más bien se debe pensar en que el campesinado tiene una cosmovisión más amplia, un horizonte más extendido sobre las estrategias de vida, y es así como poco a poco la pluriactividad es el fenómeno social que debe llamar la atención a cientistas sociales.

Esta diversidad de estrategias desarrolladas por la familia campesina, es un indicador de su adaptabilidad a las condiciones variantes del entorno, tal y como lo plantearon (Chambers \& Conway, 1991) y (Cáceres, 1995), una de las maneras de adaptarse es buscando alternativas, la búsqueda de estas, es la que permite que la cultura campesina aún perviva en el sector rural, y no lo contrario, porque aportan un ingreso extra a las UDC, sin embargo se debe mencionar nuevamente que la agricultura, sobre todo la de rubros potencialmente comercializables, tiene un peso significativo en estas comunidades. 
Revista Electrónica de Investigación en Ciencias Económicas

Facultad de Ciencias Económicas, UNAN-Managua

Estrategias de vida campesina en cuatro comunidades ubicadas en áreas protegidas: Una cosmovisión económica más allá de la agricultura

\section{Bibliografía}

Cáceres, D. (1995). Estrategias Campesinas en Sociedades Rurales

REICE $\mid 75$ Contemporáneas. Revista de la Facultad de Agronomía, 67-72.

Chambers, R., \& Conway, G. (1991). Sustainable rural livelihoods: practical concepts for the 21st century. IDS Discussion Paper, 1-27.

Chayanov, A. (1981). Sobre la teoría de los sistemas económicos no capitalistas. En J. Aricó, Chayanov y la teoría de la economía campesina (págs. 49-79). México D.F: siglo xxi editores.

Barrantes, R. (2008). Investigación un camino al conocimiento: Un enfoque cuantitativo y cualitativo. San José: EUNED.

De Grammont, H. (2008). El Concepto de Nueva Ruralidad. Bogotá: Editorial Pontificia Universidad Javeriana.

de Janvry, A., \& Sadoulet, E. (2000). Cómo Transformar en un Buen Negocio la Inversión en el Campesinado Pobre: Nuevas Perspectivas de Desarrollo Rural en América Latina. Nueva Orleans: Conferencia sobre Desarrollo de la Economía Rural y Reducción de la Pobreza en América Latina y el Caribe.

Elizalde, A. (1996). ¿Es sustentable ambientalmente el crecimiento capitalista?

Ferrus, J. (1988). Reproducción del grupo doméstico y de la explotación agrícola familiar a partir de un proceso de industrialización rural. Arxiu d'Etnografia de Catalunya, 124-134.

Fromm, E., \& Maccoby, M. (1990). Sociopsicoanálisis del campesinado mexicano. México DF: Fondo de cultura económica.

Instituto Latinoamericano y del Caribe de Planificación Económica y Social. (2012). Panorama de Desarrollo Territorial en América Latina y El Caribe. Santiago de Chile: Naciones Unidas.

Kautsky, K. (1974). La Cuestión Agraria. México: Nuestro Tiempo. 
Revista Electrónica de Investigación en Ciencias Económicas

Facultad de Ciencias Económicas, UNAN-Managua

Estrategias de vida campesina en cuatro comunidades ubicadas en áreas protegidas: Una cosmovisión económica más allá de la agricultura

Kay, C. (2007). Pobreza rural en América Latina: teorías y estrategias de desarrollo. Revista Méxicana de Sociología, 69-108.

Lanzas, C., \& Rojas, J. (2011). Estrategias de reproducción de las unidades domésticas campesinas de la comunidad Jucuapa centro, Matagalpa, Nicaragua. En J. Rojas, \& J. Ramirez, Desarrollo Rural en Nicaragua: Una visión de sus problemas y alternativas (págs. 91-113). Managua: aldea.

Meillassoux, C. (1989). Mujeres, Graneros y Capitales: economía doméstica y capitalismo. México D.F: siglo xxi editores.

Morales, O. (2003). Fundamentos de la investigación documental . Venezuela: Universidad de los Andes.

Palerm, A. (2008). Antropología y Marxismo. México DF: CIESAS.

Suárez, N., \& Tobasura, I. (2008). Lo rural: Un campo inacabado. Revista de la Facultad Nacional de Agronomía, Medellin, 4480-4495.

Teubal, M. (2001). Globalización y nueva ruralidad en América Latina. Buenos Aires: CLACSO.

Vargas, M. (1996). Estrategias de sobrevivencia, alternativas económicas y sociales de la unidad campesina. Papeles de Población, 39-50.

Wolf, E. (1971). Los campesinos. Barcelona: Labor, s.a. 